\title{
Status of the VERITAS Observatory
}

\author{
J. Holder* and V.A. Acciari, E. Aliu, T. Arlen, M. Beilicke, W. Benbow,
} S.M. Bradbury, J.H. Buckley, V. Bugaev, Y. Butt, K.L. Byrum, A. Cannon, O. Celik, A. Cesarini, L. Ciupik, Y.C.K. Chow, P. Cogan, P. Colin, W. Cui, M.K. Daniel, T. Ergin, A.D. Falcone, S.J. Fegan, J.P. Finley, G. Finnegan, P. Fortin, L.F. Fortson, A. Furniss, G.H. Gillanders, J. Grube, R. Guenette, G. Gyuk, D. Hanna, E. Hays, D. Horan, C.M. Hui, T.B. Humensky, A. Imran, P. Kaaret, N. Karlsson, M. Kertzman, D.B. Kieda, J. Kildea, A. Konopelko, H. Krawczynski, F. Krennrich, M.J. Lang, S. LeBohec, G. Maier, A. McCann, M. McCutcheon, P. Moriarty, R. Mukherjee, T. Nagai, J. Niemiec, R.A. Ong, D. Pandel, J.S. Perkins, M. Pohl, J. Quinn, K. Ragan, L.C. Reyes, P.T. Reynolds, H.J. Rose, M. Schroedter, G.H. Sembroski, A.W. Smith, D. Steele, S.P. Swordy, J.A. Toner, L. Valcarcel, V.V. Vassiliev, R. Wagner, S.P. Wakely, J.E. Ward, T.C. Weekes, A. Weinstein, R.J. White, D.A. Williams, S.A. Wissel, M. Wood, B. Zitzer ${ }^{\dagger}$

*Dept. of Physics and Astronomy and the Bartol Research Institute, University of Delaware, DE 19716, USA

$\dagger$ see http://veritas.sao.arizona.edu for a full list of affiliations

\begin{abstract}
VERITAS, an Imaging Atmospheric Cherenkov Telescope (IACT) system for gammma-ray astronomy in the $\mathrm{GeV}-\mathrm{TeV}$ range, has recently completed its first season of observations with a full array of four telescopes. A number of astrophysical gamma-ray sources have been detected, both galactic and extragalactic, including sources previously unknown at $\mathrm{TeV}$ energies. We describe the status of the array and some highlight results, and assess the technical performance, sensitivity and shower reconstruction capabilities.
\end{abstract}

Keywords: Gamma-ray telescopes PACS: $95.45 .+\mathrm{i}, 95.55 . \mathrm{Ka}$

\section{INTRODUCTION}

The current generation of $\mathrm{TeV}$ gamma-ray telescopes, H.E.S.S., MAGIC and VERITAS, provide an order of magnitude sensitivity improvement over previous instruments, and have dramatically increased the number of astrophysical gamma-ray sources, and source classes, available for study. VERITAS is the most recent of these observatories to come online, and has now completed its first full season of observations.

The VERITAS array [1] comprises four, $12 \mathrm{~m}$ diameter imaging atmospheric Cherenkov telescopes located in Tucson, Arizona $\left(31^{\circ} 40^{\prime} 30^{\prime \prime} \mathrm{N}, 110^{\circ} 57^{\prime} 07^{\prime \prime} \mathrm{W}, 1268 \mathrm{~m}\right.$ above sea level) (Figure 11). Each telescope is equipped with a pixellated photomultiplier tube (PMT) camera, providing a $\sim 3.5^{\circ}$ field of view. The array layout gives telescope baselines varying from $35 \mathrm{~m}$ to $108 \mathrm{~m}$. Construction of the array began with a prototype instrument in Fall 2003; the first full telescope was commissioned in winter 2005, and the full array completed in spring 2007.
VERITAS observations are made under clear sky conditions from September to June, with a summer shutdown due to local monsoon conditions. The 2007-2008 observing season provided $\sim 700$ hours of good, dark sky observations as well as $\sim 100$ hours taken under partial moonlight. Moonlight observations are generally made with the standard high voltage and trigger conditions, and provide a valuable increase in duty cycle: observations of the newly discovered TeV AGN WComae [2], made under moonlight, led to the detection of a flare with a flux of $\sim 25 \%$ of the Crab Nebula flux [3].

The Whipple $10 \mathrm{~m}$ telescope operates on a similar schedule at a distance of $7 \mathrm{~km}$ from the VERITAS site, and provides a trigger for high flux ( $>10 \%$ Crab flux) transient events in known TeV sources. A recent Whipple $10 \mathrm{~m}$ trigger led to the prompt measurement by VERITAS of an extreme flaring state in the TeV blazar Markarian 421, peaking at $\sim 10 \mathrm{Crab}[4]$. 


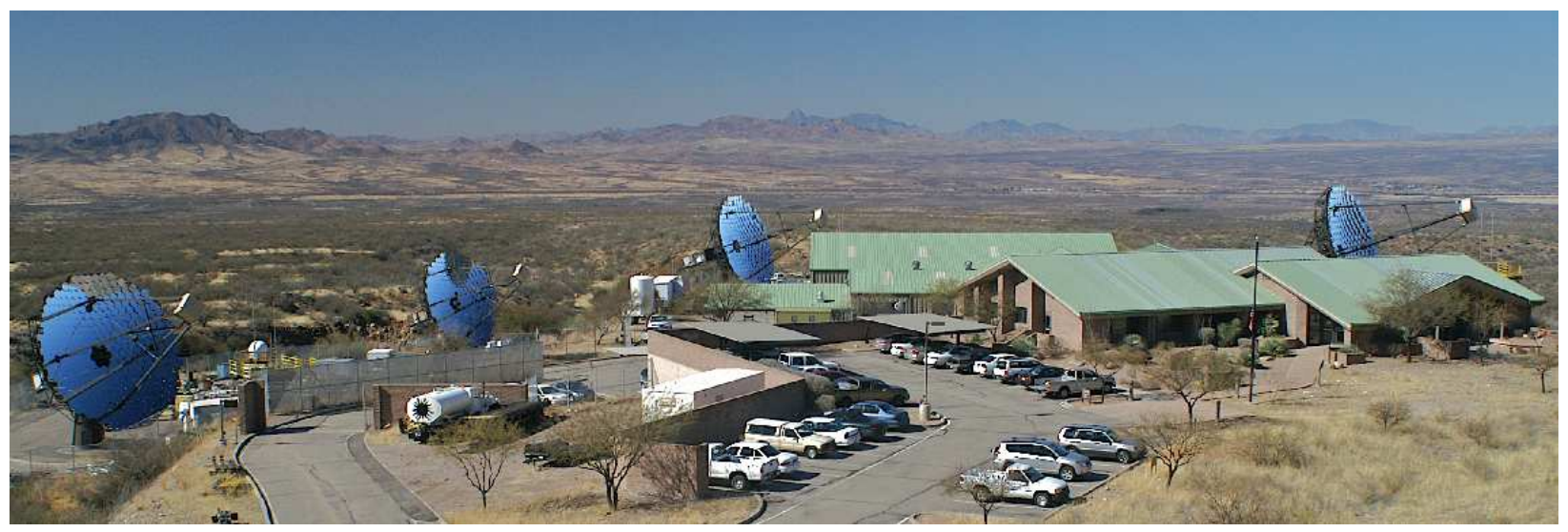

FIGURE 1. The VERITAS Array

\section{PERFORMANCE}

Each PMT pixel in the telescope cameras is instrumented with a custom-built 500 Mega-samples per second Flash ADC [5]. The FADC traces are read-out when the array trigger conditions are met (two out of four telescopes triggered, each with the signals from at least three adjacent PMTs crossing their individual constant fraction discriminator thresholds). In the first stage of the analysis, the signal traces are integrated. The resultant shower images are then calibrated, cleaned and parameterized. Gamma-ray candidate events are selected based primarily on the image shape (using "reduced mean-scaled width and length" parameters) and the reconstructed angular distance from the candidate source location, $\theta$ ([6], and references therein). The following sections present some of the critical performance metrics of the system.

\section{Angular Reconstruction}

Angular resolution is a function of both the analysis procedure and the absolute pointing accuracy of the telescopes in the array. Calibration of the telescope pointing is performed on a monthly basis, and the results input to a pointing model in the telescope tracking software which corrects for the measured misalignments and flexures during observations. This provides a systematic pointing accuracy of $\sim 0.02^{\circ}$. Recently installed optical pointing monitor telescopes provide further improvement offline.

The optimum angular resolution and sensitivity to weak sources is achieved when relatively strict gammaray selection cuts are applied. The results shown here require that all four telescopes are involved in the shower reconstruction, and that each image has an integrated charge (size) per telescope of $>75$ photo-electrons. With these requirements we obtain the energy dependent angular resolution illustrated in Figure 2] Averaged over all energies, the angular resolution for a single event is $\sim 0.1^{\circ}$ (68\% containment radius). This source localization capability allows us to distinguish between point sources and sources with a small angular extent, as illustrated by VERITAS observations of the extended TeV source in the IC443 supernova remnant [8].

\section{Sensitivity}

Using the same strict cuts, with the addition of an angular cut of $\theta<0.12^{\circ}$, Figure 3 illustrates the current sensitivity of the array as measured using Crab Nebula observations. An unresolved source with a flux of $1 \%$ that of the Crab Nebula is detected in 47 hours of observations, a 5\% Crab source requires 2.5 hours. The TeV blazar 1ES $0806+524$ has been detected by VERITAS at this flux level [9]. Figure 3 also shows the gammaray rate as a function of the source position in the field of view. The array has useful sensitivity out to at least $2.0^{\circ}$, which has allowed the measurement of a gammaray flux from 1ES $1218+304$ during observations centered around WComae, separated by $1.94^{\circ}$ [2]

\section{Energy Reconstruction}

The energy of incident gamma rays is calculated from the measured impact parameter, image size, and azimuth and elevation angle using lookup tables filled from simulated gamma-ray events. The energy scale of the simulations is calibrated using a variety of complementary methods, such as in situ measurements of the PMT single photo-electron response, studies of the light yield from local muons, and measurements of the Rayleigh scattered light from a nearby laser pulse [10]. In order to provide a wide dynamic range for the energy spectra, gammaray selection cuts are loosened to allow any event with at least two telescopes recording an image with $>4$ pix- 

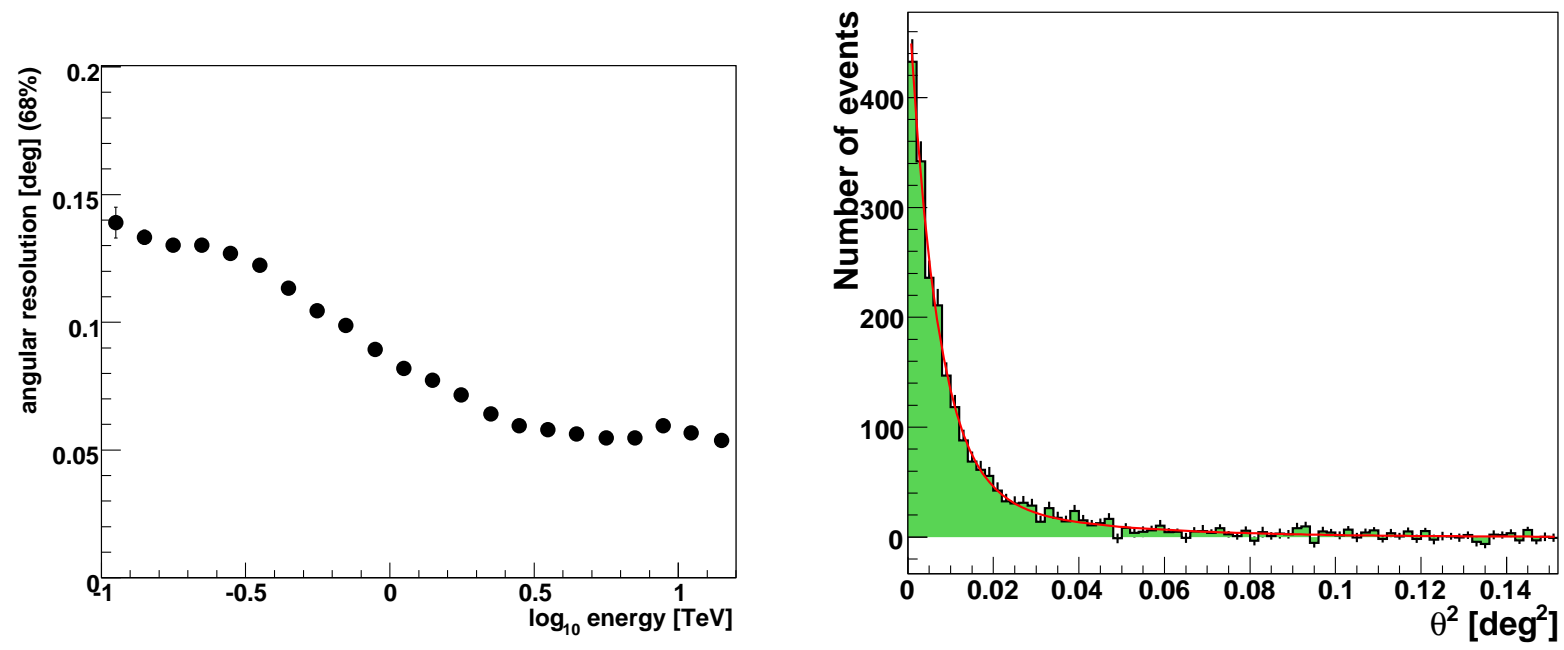

FIGURE 2. Left: The angular resolution as a function of energy, measured using simulated gamma-ray events. Right: The $\theta^{2}$ distribution for the gamma-ray excess measured during observations of the Crab Nebula. The fit is a combination of two Gaussian functions, as described in [7]
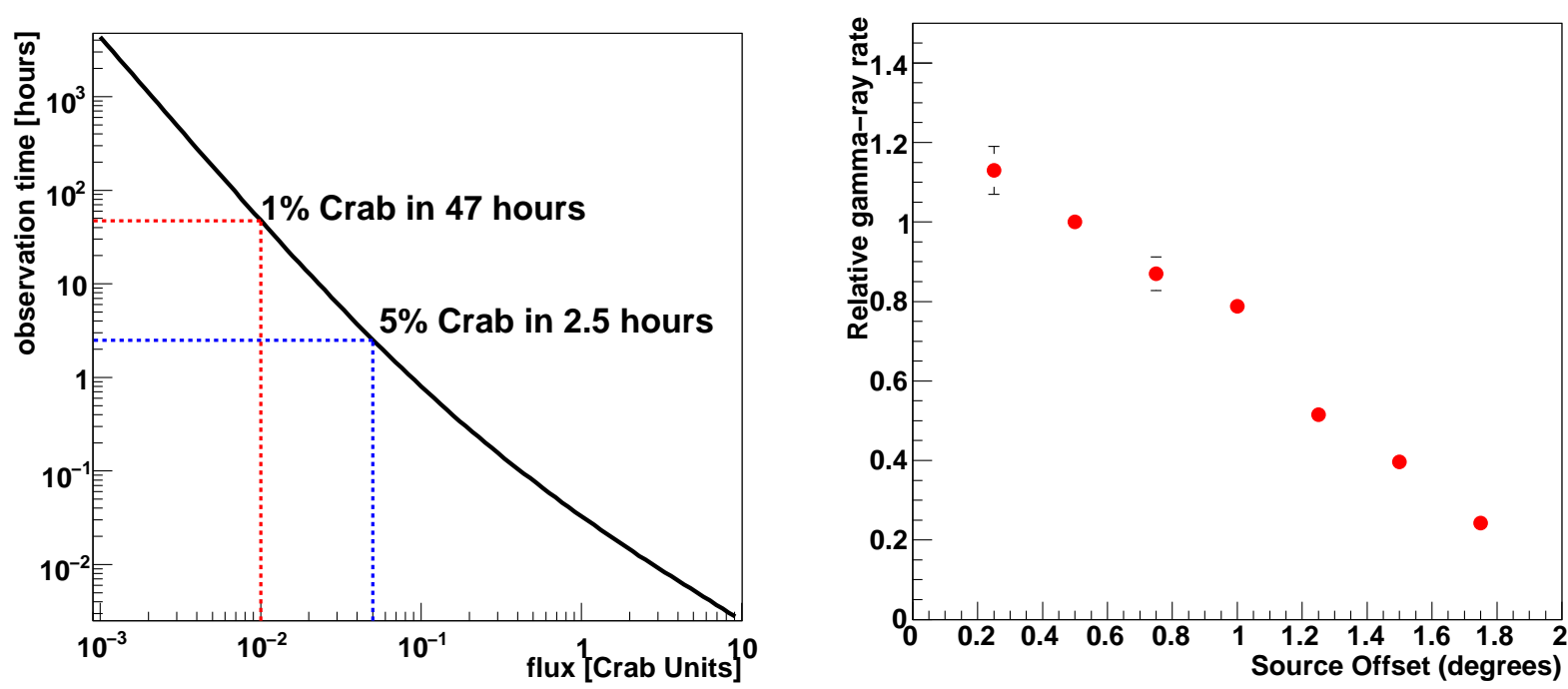

FIGURE 3. Left: The time required for a $5 \sigma$ detection for different source strengths, assuming a Crab-like gamma-ray spectrum. Right: The gamma-ray rate as a function of source position, measured using observations of Crab Nebula. The measurements are normalized to the measured rate at an offset of $0.5^{\circ}$. 


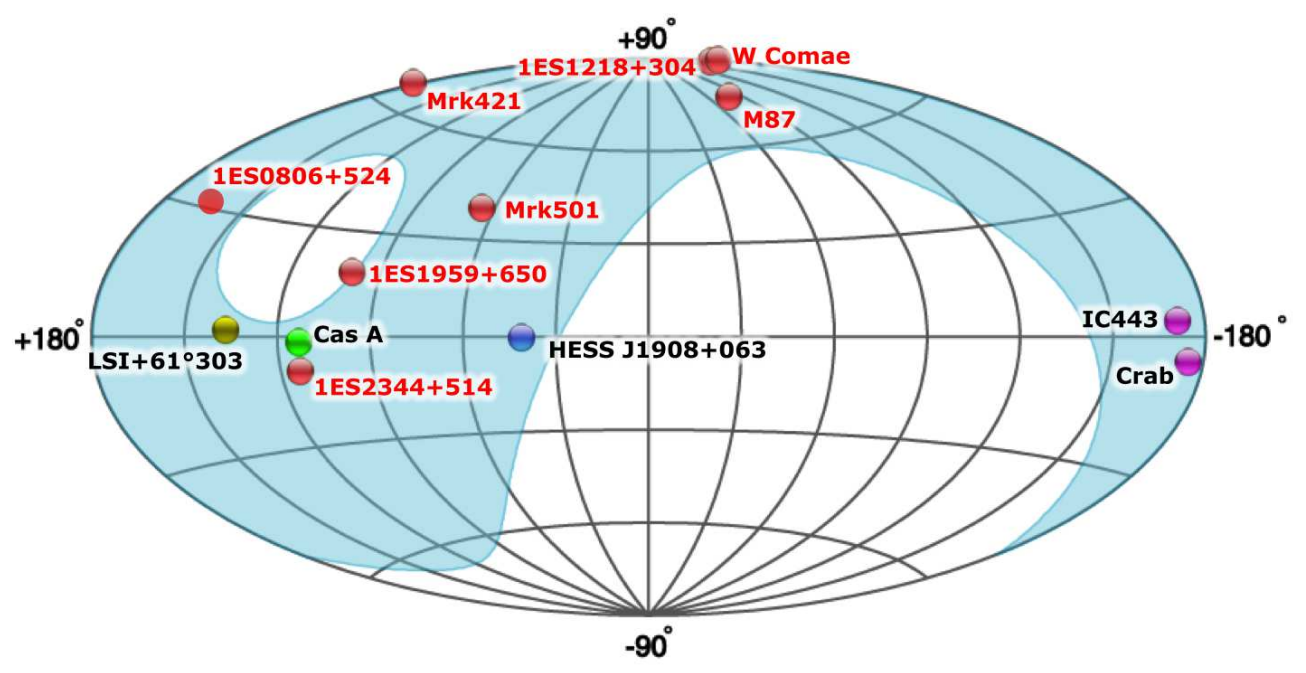

FIGURE 4. The VERITAS source catalogue as of summer 2008 (in galactic coordinates). Extragalactic sources are labelled in red, those within our galaxy are labelled in black. The shaded area shows the region of the sky visible to VERITAS at elevations greater than $55^{\circ}$. Figure provided courtesy of TevCat (http: //tevcat.uchicago.edu/)

els, excluding the telescope combination with the smallest baseline (Telescopes 1 and 4). Spectral reconstruction is possible from a minimum energy of $\sim 150 \mathrm{GeV}$ and with an energy resolution of $\sim 15 \%$ at high energies.

\section{SUMMARY}

We have completed the first full season of observations with the four-telescope VERITAS array. The technical performance, site conditions and mechanical reliability are good, providing $\sim 800$ hours of clear sky observations each year. Highlight results from this first season include the detection of two new TeV sources, 1ES 0806+524 and WComae, and detailed studies of the supernova remnants IC443 and Cas A, including a measurement of an extended $\mathrm{TeV}$ source in IC443 [8]. Extensive multiwavelength observations have been made of known extragalactic $\mathrm{TeV}$ sources, including a comprehensive multi-instrument campaign on M87 [11] the detection of extreme flaring from Markarian 421 and the highest recorded flux from 1ES 2344+514 [9]. Additional observations include uniquely interesting galactic sources such as the $\mathrm{TeV}$ binary LS I+61 ${ }^{\circ} 303$ [12] and HESS J1908+063 [13], identified with the Milagro source MGRO J1908+06.

We conclude by presenting the VERITAS source catalogue at the close of this first season (Figure 4), containing 13 northern hemisphere $\mathrm{TeV}$ sources. Ongoing projects include a survey of the Cygnus region of the galactic plane, and a search for gamma-ray signatures from objects predicted to have an enhanced dark matter component.

\section{ACKNOWLEDGMENTS}

This research is supported by grants from the U.S. Department of Energy, the U.S. National Science Foundation, the Smithsonian Institution, by NSERC in Canada, PPARC in the U.K. and by Science Foundation, Ireland.

\section{REFERENCES}

1. Holder, J., et al. Astropart. Phys., 25, 391 (2006).

2. Acciari, V.A. et al (The VERITAS Collaboration), ApJ, 648, L73 (2008).

3. Swordy, S. et al (The VERITAS Collaboration), Astronomer's Telegram \#1565 (2008).

4. Reyes, L. et al (The VERITAS Collaboration), These proceedings (2008).

5. Buckley, J.H. et al. Proc. 28th ICRC, Tsukuba, Eds: T. Kajita, Y. Asaoka, A. Kawachi, Y. Matsubara and M. Sasaki, 2827 (2003).

6. Acciari, V.A. et al (The VERITAS Collaboration), ApJ, 679, 1427 (2008).

7. Lemoine-Goumard, M., Degrange, B. \& Tluczykont, M., Astropart. Phys. 25, 195 (2006).

8. Humensky, T.B. et al (The VERITAS Collaboration), These proceedings (2008).

9. Cogan, P. (The VERITAS Collaboration) These proceedings (2008).

10. Hui, C.M. et al (The VERITAS Collaboration), These proceedings (2008).

11. Beilicke, M. (The VERITAS, MAGIC and H.E.S.S. Collaborations) These proceedings (2008).

12. Maier, G. (The VERITAS Collaboration) These proceedings (2008).

13. Ward, J.E. (The VERITAS Collaboration) These proceedings (2008). 